\title{
The Importance of Further Studies and Missions to Understand Cryovolcanism
}

Co-authors: Catherine C. Walker ${ }^{1}$, Kelsi N. Singer ${ }^{2}$, Kate L. Craft ${ }^{3}$, Marc Neveu ${ }^{4,5}$, Ross A. Beyer ${ }^{6,7}$ Michael Sori $^{8}$, Conor A. Nixon ${ }^{4}$, Jennifer Scully ${ }^{9}$, Julie CastilloRogez $^{9}$, Morgan L. Cable ${ }^{9}$, Francis Nimmo ${ }^{10}$, Sarah Fagents $^{11}$, Rosaly M. Lopes ${ }^{9}$, Terry Hurford $^{4}$, Jessica L. Noviello ${ }^{12}$, Debra Buczkowski ${ }^{3}$, Shannon MacKenzie ${ }^{3}$, Paul K. Byrne $^{24}$

Co-signers: Linda Spilker ${ }^{9}$, Louise Prockter $^{18}$, Oliver White ${ }^{6}$, Sarah Waller ${ }^{9}$, Heather Meyer $^{3}$, Mark Gurwell ${ }^{13}$, James T. Keane ${ }^{9}$, Adeene Denton ${ }^{8}$, Laurent Montesi ${ }^{5}$, Richard J. Cartwright ${ }^{6}$, Jorge I. Núñez ${ }^{3}$, Emily K. Hawkins ${ }^{14}$, Andrew J. Dombard ${ }^{15}$, Tanguy Bertrand $^{7}$, David A. Williams ${ }^{12}$, Charity Phillips-Lander ${ }^{2}$, Tim I. Michaels ${ }^{6}$, Maheenuz Zaman $^{16}$, James Haber ${ }^{8}$, Chloe Beddingfield ${ }^{4,6}$, Jonathan P. Kay ${ }^{17}$, Cynthia B. Phillips ${ }^{9}$, Paul Schenk ${ }^{18}$, Jennifer G. Blank ${ }^{7,19}$, Miki Nakajima ${ }^{20}$, Edwin S. Kite ${ }^{21}$, Rutu Parekh ${ }^{22}$, Michele T. Bannister ${ }^{23}$, Scott L. Murchie ${ }^{3}$, Joshua T.S. Cahill ${ }^{3}$, Kathleen E. Mandt ${ }^{3}$, Michael Manga ${ }^{25}$, Robert T. Pappalardo ${ }^{9}$, D. Alex Patthoff ${ }^{26}$, Lauren M. Jozwiak ${ }^{3}$, Michael J. Malaska ${ }^{9}$, M. Alexandra Matiella Novak ${ }^{3}$, Abigail M. Rymer ${ }^{3}$, Mathieu Choukroun $^{9}$, Mallory J. Kinczyk ${ }^{24}$, Jenny Kampmeier ${ }^{9}$, Danielle Wyrick ${ }^{2}$, Veronica Bray $^{27}$, Orkan M. Umurhan ${ }^{6,7}$, Jeffrey M. Moore ${ }^{7}$, Marissa E. Cameron ${ }^{9}$, Alfred S. McEwen $^{27}$, Jennifer Hanley ${ }^{28}$, Lauren Schurmeier ${ }^{11}$, Will Grundy ${ }^{28}$, Alice Lucchetti ${ }^{29}$, Maurizio Pajola ${ }^{29}$, Carol Paty ${ }^{30}$, Erin Leonard ${ }^{9}$, Catherine Elder ${ }^{9}$, Alan Howard ${ }^{26}$, Olga Prieto-Ballesteros ${ }^{31}$, Rosemary A. Millham ${ }^{32}$, Kirby Runyon ${ }^{3}$, Rudi Lien ${ }^{33}$, Graham Lau $^{19}$, Everett L. Shock ${ }^{12}$, Divya M. Persaud ${ }^{34}$, Krista M. Soderlund ${ }^{35}$, Carey M. Lisse ${ }^{3}$, Eric S. Boyd ${ }^{33}$, Aaron Morrison ${ }^{36}$, Vinayak Shastri ${ }^{37}$

Affiliations: ${ }^{1}$ Woods Hole Oceanographic Institution, ${ }^{2}$ Southwest Research Institute, ${ }^{3}$ Johns Hopkins University Applied Physics Laboratory, ${ }^{4}$ NASA Goddard Space Flight Center, ${ }^{5}$ University of Maryland, ${ }^{6}$ SETI Institute, ${ }^{7}$ NASA Ames Research Center, ${ }^{8}$ Purdue University, ${ }^{9}$ Jet Propulsion Laboratory, California Institute of Technology ${ }^{10}$ University of California Santa Cruz, ${ }^{11}$ University of Hawai 'i at Mānoa, ${ }^{12}$ Arizona State University, ${ }^{13}$ Center for Astrophysics | Harvard \& Smithsonian, ${ }^{14}$ Loyola Marymount University, ${ }^{15}$ University of Illinois at Chicago, ${ }^{16}$ Lamont-Doherty Earth Observatory, Columbia University, ${ }^{17}$ The College of William \& Mary, ${ }^{18}$ Lunar and Planetary Institute, ${ }^{19}$ Blue Marble Space Institute of Science, ${ }^{20}$ University of Rochester, ${ }^{21}$ University of Chicago, ${ }^{22}$ DLR Institute of planetary science, Berlin, ${ }^{23}$ University of Canterbury, NZ,

${ }^{24}$ North Carolina State University, ${ }^{25}$ University of California, Berkeley, ${ }^{26}$ Planetary Science Institute, ${ }^{27}$ University of Arizona, ${ }^{28}$ Lowell Observatory, ${ }^{29}$ INAF-OAPD, Italy, ${ }^{30}$ University of Oregon, ${ }^{31}$ Centro de Astrobiologia-CSIC-INTA, ${ }^{32}$ SUNY New Paltz, ${ }^{33}$ Montana State University, ${ }^{34}$ University College London, ${ }^{35}$ University of Texas at Austin, ${ }^{36}$ University of Texas San Antonio, ${ }^{37}$ University of St Andrews 


\section{Executive Summary}

\section{Studying cryovolcanism is critical to understanding the geologic history and activity of icy worlds in the Solar System and has the potential to enhance studies of habitability and enable detections of biosignatures.}

Cryovolcanism, the extrusion of cold, fluidized material from within a planetary body, is an enigmatic and important mechanism that can enable subsurface-surface material exchange on ice-covered ocean worlds (those with extant or relict oceans). This process, therefore, has implications for surface morphology formation, understanding movement of material through ice shells, the potential for providing near-surface access to biosignatures and signatures of habitability from a subsurface ocean, and may also be a source of plume activity.

We recommend support of additional laboratory, modeling, and analog studies through R\&A funding to increase understanding of cryovolcanic processes. We also suggest future missions should target regions of cryovolcanism to collect data on past and recent activity, to increase our understanding of ice-covered ocean worlds and the distribution of life in the

\section{Background}

Cryovolcanism is a term used to represent the mechanism that causes fluid/icy material in the subsurface of ice-covered ocean worlds to ascent towards and possibly erupt onto the surface. The fluid/icy material, herein called “cryomagma," could be pure water, a salty fluid, and/or partially frozen material with additional chemical compounds. As described by Geissler ${ }^{1}$, cryovolcanism is “... the eruption of liquid or vapor phases (with or without entrained solids) of water or other volatiles that would be frozen solid at the normal temperature of the icy satellite's surface." Cryovolcanic processes have important similarities and differences to those of silicate volcanism ( ${ }^{{ }^{2}}$ Kerber et al. WP). Magma ascent and landform formation are in some ways qualitatively similar in both cases, but the different chemistry, density, and rheology of cryomagma and the surrounding layers' composition demands that careful numerical and laboratory experiments be considered when interpreting potential cryovolcanic activity. For cases where the overlying crust is less dense than the cryomagma, it is theorized the cryomagma can move towards the surface driven by a pressure gradient from, e.g., a cooling and thickening ice shell or a cooling cryomagma chamber ${ }^{3}$, along with potential release of volatiles aiding the cryomagma's rise ${ }^{4}$.

\section{Importance of Cryovolcanism}

A. Ocean/subsurface reservoir-to-surface exchange

\section{Subsurface}

As a mechanism that potentially brings icy material to the surface from the ocean or subsurface reservoirs, cryovolcanism is extremely important in that it may enable the sampling of relatively young ocean/subsurface material. Numerous studies have investigated the potential of subsurface fracturing and pressure gradients that enable fluid to travel from an ocean or subsurface reservoir towards the surface. A cooling and thickening ice shell overlying an ocean may pressurize the ocean such that when a fracture opens due to internal stress ${ }^{5,6}$ or external mechanisms (e.g., impacts), the fluid can ascend towards the surface ${ }^{4,7}$, potentially assisted by the release of volatiles ${ }^{8-11}$ and/or by tidal forces acting on fractures ${ }^{12-14}$. Additionally, 
reservoirs that form within the icy crust $^{7,15-17}$ can cool and start to pressurize causing cryomagmatic dikes to form and propagate towards the surface ${ }^{18-22}$. Recently, some attention has been paid to the separation of melt from solids (compaction or two-phase flow), which can lead to chemical fractionation, traces of which might be detectable at the surface ${ }^{23,24}$. However, even with these studies, the data needed to validate the models and experiments remain to be obtained. To fully understand cryovolcanism, we must send more missions to targets with likely cryovolcanic features, complemented by additional experiments and modeling.

\section{Geologic surface feature and morphology formation mechanisms}

Because in situ observations from landed spacecraft on icy bodies are a rarity and have yet to access any icy subsurface ( ${ }^{2} 25$ Schmidt et al. WP) we depend upon remote observations. Surface features become the lens through which we are able to interpret subsurface structures and processes. Features interpreted as manifestations of cryovolcanic activity have been identified on several bodies remotely from orbit or during flybys. Examples include grooved terrain and caldera-like features on Ganymede ${ }^{26,27}$; plumes of water and ice from Enceladus' south polar fractured terrain ${ }^{28}$, plumes emanating from nitrogen-rich areas on Triton $^{29}$, and putative water plumes on Europa ${ }^{30-32}$; flow features on moons like Ariel and Miranda ${ }^{33}$; flows, channels and other putative cryovolcanic constructs on Titan ${ }^{34-37}$; floorfractured crater morphologies and domed edifices on dwarf planet Ceres $^{37}$; ridges, pits, domes, and chaos terrain across the surface of Europa ${ }^{15,39,40}$; and exotic annular massifs on Pluto ${ }^{41}$. Some of these are detailed below.

The broad range of planetary surfaces, the subsurfaces they hide, and such exemplary surface features highlight the fact that the geomorphology of landforms associated with putative cryovolcanic processes often represent some of the most striking features against their respective planetary surface backdrops, possibly developing from a variety of subsurface processes, activities, and structures. Some possible subsurface mechanisms involved are changing stress states (driven internally or externally), fracturing, flow of liquid water or other volatile material, freezing or state change of that material, and associated density differences in those materials that overcome (or not) the confining pressure in the near-surface. Surface features associated with cryovolcanism can occur not only at a variety of spatial scales, but also across a range of timescales, from instantaneous to hours to days (e.g., plumes) to years to thousands of years (e.g., mountain building). Because of the wide assortment of cryovolcanic processes/expressions and the time and spatial scales over which they operate, it is essential to collect both short- and long-term observations for a broad range of features and bodies to improve understanding of where, how, and why cryovolcanism occurs in our Solar System, and importantly, what it indicates is occurring/has occurred beneath the surface.

\section{Possible driver for plumes/re-surfacing activity}

Atmospheric or above-surface events, such as constant or transient plumes, can also result from reservoir-surface fluid/gas exchange. Such processes that involve the near-surface and above-surface are influential in terms of resurfacing activity and, relatedly, our ability to determine not only surface age but also timescales of active processes.

\section{B. Heat flow processes and thermal history of body}

Another way cryovolcanic features can reveal information about the interior of a body is by recording the thermal history and stress states at the time and location of feature 
formation. To have mobile material, a material-dependent threshold thermal state and driving pressure gradient of some kind is assumed to be needed. If other information, such as the age of the features is available (e.g., estimated from the superposition of other features or from crater measurements), cryovolcanic features can help understand the local heat flux and stress state over time on that body. The variety of potential cryovolcanic features on different icy bodies (see below) requires that our field be creative about the conditions and materials that allow material movement on and within these bodies, as some of these features appear surprisingly young and may have formed even in thick ice shells.

\section{Access to biosignatures}

Cryovolcanism may provide access to biosignatures that might otherwise be challenging to reach. Biosignatures are chemical or structural evidence of past or present life, and may include isotopes/molecules or their patterns (i.e., the ratio of amino acids relative to glycine), cells or cell motility, and active or fossilized microbial mats (e.g., stromatolites) or other biofilms/biominerals. Many of these biosignatures could be present in the subsurface reservoir of an ocean world, and through cryovolcanism be expressed onto the surface or into space via a plume (such as at Enceladus). More experimental and modeling work is needed to understand how such biosignatures may be processed or altered during the cryovolcanism process (via pressure, temperature, etc.). Additionally, some mechanisms ${ }^{42}$ (i.e., formation of briny pockets, or bubble scrubbing) might spatially concentrate biosignature molecules and thus improve the likelihood of detection at the surface by a lander or an orbiter.

\section{Potential Cryovolcanic Activity in the Solar System (selected features): Europa}

Jupiter's icy moon Europa is a compelling target for future habitability studies mainly because of its confirmed global, salty liquid water ocean ${ }^{43}$, which lies underneath a relatively thin $^{44}(<30 \mathrm{~km})$ ice shell. Europa's surface geology is a direct manifestation of its subsurface activity, and therefore its ocean. Double ridges, the most ubiquitous landform on Europa, may originate as cryovolcanic fissure eruptions ${ }^{15}$. Europa displays a myriad of features with varying combinations of uplifts, pits, and chaotic-style fractures/breakup, all of which may be related to intrusions and/or extrusions onto the surface ${ }^{45}$. Certain domes and dome-like features on Europa have been included as evidence of cryovolcanism ${ }^{8}{ }^{46}$, along with discoloration on surface bands and ridges ${ }^{47}$. Microchaoses, and chaos terrain in general, may also be related to a more effusive type of cryovolcanism. Chaos terrain is thought to be formed by localized heating and melting of surface or near-surface ice, followed by a brief period where ice fragments are either completely melted or mobilized, and then "refreeze" into their present appearances ${ }^{16,48,49}$ (image of Murias

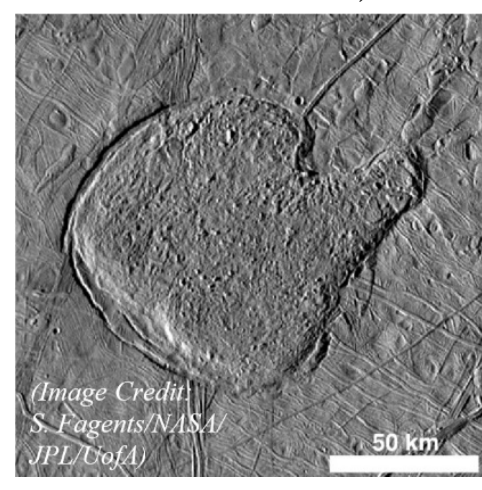

Chaos at left). Often, but not always, chaos terrain is also associated with areas of low albedo ${ }^{50}$ and a higher concentration of salts $^{51}$, suggesting a connection to the salty subsurface ocean. Thus, chaos terrain may be considered cryovolcanic because of its relationship to heat and material transport within Europa's ice shell. Observations ${ }^{30-32}$ of possible plume activity may also point to cryovolcanic activity at Europa, although such activity has yet to be confirmed. Finally, "hybrid" features, showing a combination of topographic expressions, albedo patches, and various degrees of fracturing, may also be associated with cryovolcanism ${ }^{7,17}$. 


\section{Enceladus}

Enceladus presents the best-known example of active cryovolcanism: individual jets of water vapor and salty ice crystals emanating from fractures (like the ones shown at right) at the south pole $^{28}$. These jets provide samples of a subsurface ocean, which were analyzed in situ by the Cassini spacecraft ${ }^{52}$ and could in principle be captured and/or returned to Earth for further study $\left({ }^{5} 53 \mathrm{Neveu}\right.$ et al. WP). The jets merge to form a plume, which varies in strength over an individual orbital cycle ${ }^{54}$ and also on multi-year timescales ${ }^{55}$. This periodic diurnal behavior is almost certainly due to diurnal tidal stresses causing the opening and closing of fractures ${ }^{56}$; the mechanism for the longer-period behavior is still under investigation.

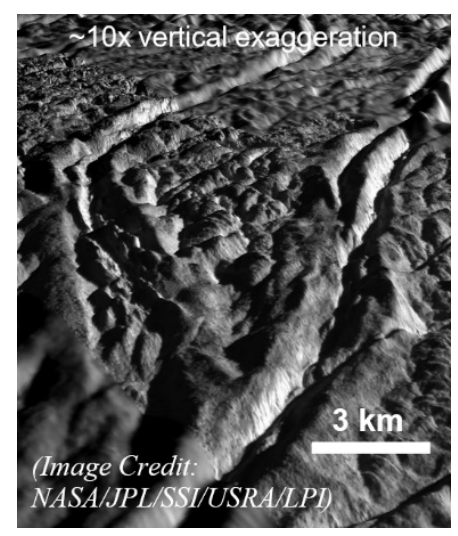

The material in the enceladan plumes likely represents the composition of the subsurface ocean with very little alteration as material is transported through its ice shell. As evidence to this fact, Cassini's Cosmic Dust Analyzer instrument detected silica nanoparticles in the E-ring, which is sourced from Enceladus' plumes; these particles are linked to ongoing, hydrothermal activity from Enceladus' sub-surface ocean floor ${ }^{57}$. Indeed, models of tidal stresses on Enceladus conservatively estimate magnitudes of $100 \mathrm{~s}$ of $\mathrm{kPa}$, which can drive active fractures to depths of up to a few kilometers ${ }^{56}$. And estimates of ice shell thicknesses ranging from a few to $10 \mathrm{~s}$ of $\mathrm{km}$ imply that subsurface ocean water can infiltrate to within a few kilometers of the surface, where tidally active fractures can interact with this material. Although Enceladus emits significant excess heat at the south pole $\mathrm{e}^{58}$, little is advected by the erupting plume material: the majority of the heat transfer probably arises from the cooling of water in near-surface cracks, either by conduction or via condensation of vapor on the crack walls ${ }^{59}$.

\section{Titan}

Cryovolcanic structures on Titan are still the subject of some controversy, which cannot be definitively resolved by the spatial resolution and coverage of Cassini data ${ }^{60}$. The concentration of $\mathrm{Ar}^{40}$ in Titan's atmosphere detected by the Huygens probe suggests that the atmosphere communicates with a reservoir of the parent nuclide. If this reservoir is the subsurface ocean, then the ocean and atmosphere are in communication via the icy shell, and the upwelling of large cryovolcanic plumes may be releasing sufficient methane into Titan's atmosphere to account for the present atmospheric composition ${ }^{61}$. Several flow-like surface features have been identified on the surface $^{34}$ but a cryovolcanic origin has been disputed $^{62}$. The strongest surface evidence of cryovolcanic activity is an equatorial region where Sotra Patera, a non-circular, $>1 \mathrm{~km}$ deep pit, lies adjacent to a tall ( $>1 \mathrm{~km}$ high) mountain, Doom Mons (perspective view at right), from which lobate flowlike features emerge ${ }^{36}$. Spectral analysis ${ }^{63}$ suggests temporal variations in brightness in the Sotra Patera

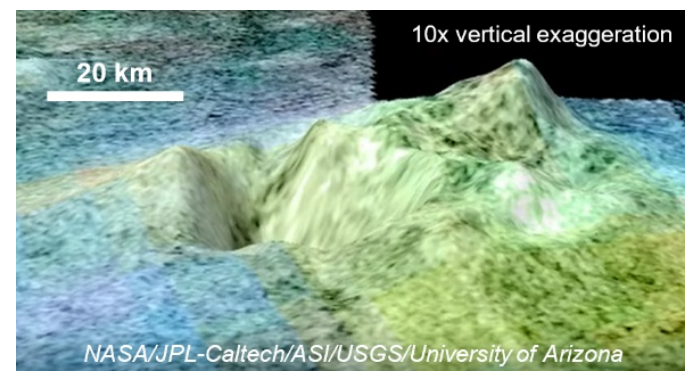
region. Cryovolcanism has also recently been suggested as a lake-basin forming mechanism at Titan's north pole ${ }^{37}$. Furthermore, the transient liquid water environments created by extruded lava flows offer the unique opportunity to investigate the evolution of prebiotic chemistry on Titan's surface ${ }^{64}$ and perhaps even the composition and habitability of the deep subsurface ocean. Determining whether Titan is or has been cryovolcanically active is an important, open 
question, the answer to which has implications for the longevity of the atmosphere, the evolution of the surface, the likelihood of surface-subsurface exchange, and the timescales of prebiotic chemistry at Titan.

\section{Triton}

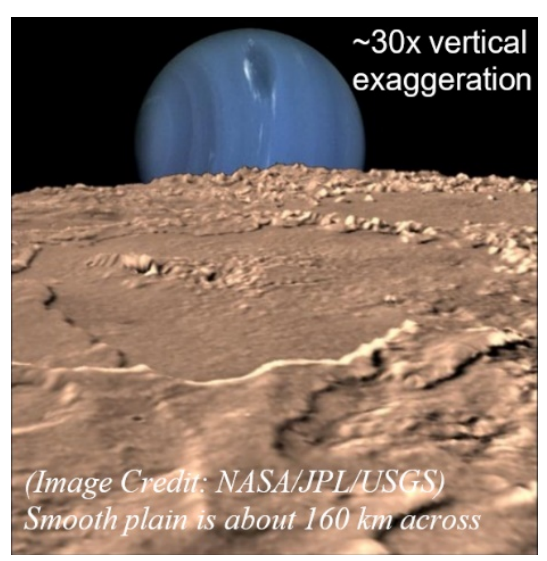

Neptune's moon Triton is thought to be a captured Kuiper belt object, and exhibits a young surface with a fascinating array of features, many of which appear to be unique to Triton $^{65}$. Like other bodies discussed here, the origin of many of these features, the source of internal heat flux that caused resurfacing of Triton-and whether Triton has an extant ocean-is not well constrained; more data are needed. Smooth terrains with central structures reminiscent of calderas (image at left) may be indicative of low-viscosity icy lava extruding onto, and flooding, the surface. Large pit chains resemble volcanic collapse chains ${ }^{66}$. It is not known how the visually striking plumes on Triton are generated, with both subsurface greenhouse effects and possible cryovolcanic origins proposed ${ }^{29,67}$. The cantaloupe terrain may be generated by diapirism ${ }^{68}$, which, if extruded onto the surface, could be a solid-state (or potentially partially molten) material emplacement and resurfacing ( ${ }^{{ }^{6} 69}$ Hansen et al.WP).

\section{Pluto, Charon}

Possible volcanic landforms on Pluto are diverse. Wright (perspective view below) and Piccard Montes are two large annular massifs with hummocky flanks and few-to-no craters ${ }^{41,70}$. They appear to be constructional (rather than erosional remnants) and could involve large volumes of (likely viscous) material emplacement onto the surface. Other expressions of cryovolcanism on Pluto may include a much less viscous effusion and flow of a water-ammonia mix into extensional faults west of Sputnik Planitia, in which organic material has optical properties that may result from chemical processing inside Pluto ${ }^{71,72}$. On Pluto, magma extrusion by pressurizing an ocean is more difficult due to higher overburden pressure ${ }^{73,74}$. Cryovolcanism may have been facilitated by stress induced by the reorientation of Pluto ${ }^{75,76}$ and/or by surface loading from condensing $\mathrm{N}_{2}{ }^{71}$ that may have opened fractures through which fluids ascended by a combination of pressurization and buoyancy. The latter process may be enhanced by the exsolution of dissolved gases, especially those that do not readily dissolve in $\mathrm{H}_{2} \mathrm{O}$ such as $\mathrm{H}_{2}, \mathrm{~N}_{2}$, and $\mathrm{CH}_{4}{ }^{11}$.

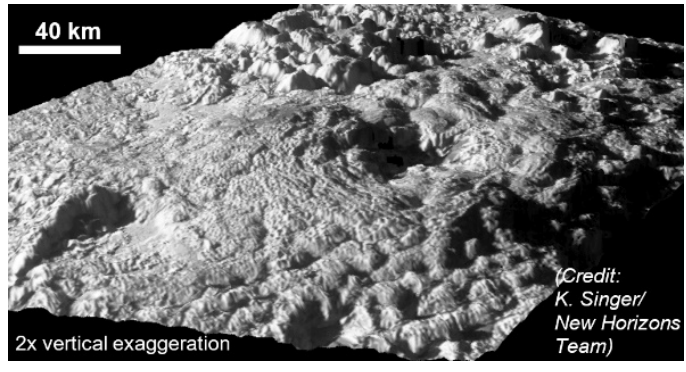

On Charon, the large, smooth plains of Vulcan Planitia suggest widespread resurfacing by flowing material, likely a water-ammonia mixture once more ${ }^{77}$. Within these plains, intriguing moated mountains may be embayed lithospheric blocks ${ }^{\text {Ibid. }}$ or the expression of subsequent volcanic effusion and flexure of a thin lithosphere above local liquid reservoirs undergoing freezing $^{78}$, as has been postulated to occur on Ceres. Cryovolcanism on Charon likely arose from global expansion from secular cooling (whereby heat transport exceeded heat production by radioactivity, and early on by impacts and tidal dissipation). Also see Holler et al. ${ }^{7}{ }^{79}$, Robbins et al. ${ }^{880}$, Howett et al..$^{881}$, and Runyon et al. ${ }^{882}$ WPs on Pluto-system and KBO exploration. 


\section{Ceres}

Ceres, the largest object in the asteroid belt and most accessible ice-rich body in the Solar System, has been revealed by NASA's Dawn mission ${ }^{83,884}$ (Castillo-Rogez et al. WP) to likely be a cryovolcanic world. Dawn observations revealed three classes of surface features ${ }^{37}$ on its icy/salty/rocky crust that are plausibly formed by cryovolcanic extrusions: bright faculae within impact craters $^{85}$, floor fractured craters, and isolated mountains that do not appear to be connected to impacts ${ }^{86}$.

Faculae are anomalously bright features, probably of evaporitic nature and derived from a subsurface brine source. They are composed mostly of carbonates and chlorides ${ }^{87,88}$, and some are young ( $\sim$ Myrs old) indicating geologically recent activity ${ }^{89}$. An endogenic source for these young brines is hypothesized to be the remnants of an ancient Cerean subsurface ocean rising through fractures ${ }^{21,90}$.

Isolated mountains across Ceres may also be cryovolcanic, analogous to extrusive lava domes. The most prominent example is Ahuna Mons (image at right), a 4-km-tall mountain that is geologically young (<100s of Myrs old) and hypothesized to result from extrusions sourced from a "muddy" mantle ${ }^{91}$. Other more subdued mountains may represent older, modified cryovolcanic edifices ${ }^{86}$, which have been used to estimate an average cryovolcanic eruption rate on Ceres of $\sim 10^{4} \mathrm{~m}^{3} / \mathrm{yr}$. An alternative hypothesis to the cryovolcanic interpretation is that the mountains are instead formed by diapirism, analogous to salt tectonic processes on Earth ${ }^{92}$. High-resolution $(<5 \mathrm{~m} / \mathrm{pix})$ visible and color imaging and gravity ( $<20 \mathrm{~km}$ half wavelength) data are required to test these two hypotheses and more fully characterize cryovolcanism on Ceres.

Lastly, several of the impact craters on Ceres display patterns of fractures on their floors, including Occator $^{90}$, that appear similar to those on both the Moon and Mars. These features, referred to as "floor-

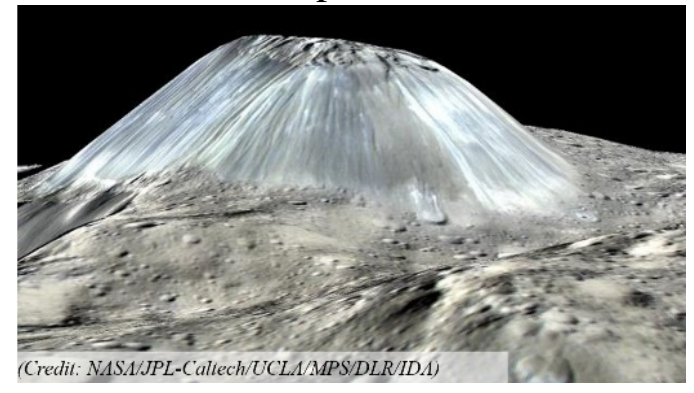
fractured craters" (FFCs), are thought to experience floor uplift due to magmatic intrusion below the crater ${ }^{93,94}$. Evaluation of the FFCs on Ceres showed that they are likely also undergoing fracturing because of the intrusion of a low-density material, likely cryomagma, below the craters $^{90}$.

Please note: We recommend that the decadal survey consider the critical role of team dynamics, equity, diversity, inclusion, and accessibility in planetary science. [Please see

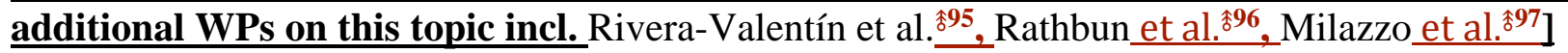

Citations: Please note that there are many additional references we were not able to include due to space restrictions. Cryovolcanism is a growing field of interest and we encourage the reader to explore references cited within those stated here. $\hat{\delta}=2020$ Decadal Survey white paper

${ }^{1}$ Geissler (2015), 10.1016/B978-0-12-385938-9.00044-4; ${ }^{2}{ }^{2}$ Kerber+ (2020), The Importance of Planetary Volcanism and Key Investigations for the Next Decade; ${ }^{3}$ McGovern+ (2018), Cryovolcanism in the Solar System Workshop; ${ }^{4}$ Manga + (2007), 10.1029/2007GL029297; ${ }^{5} \mathrm{Nimmo}$ (2004), 10.1029/2004JE002347; ${ }^{6} \mathrm{Mitri}+$ (2008), 10.1016/j.icarus.2008.02.024; ${ }^{7} \mathrm{Craft}+$ (2016), 10.1016/j.icarus.2016.01.023; ${ }^{8}$ Fagents (2003), 10.1029/2003JE002128; ${ }^{9}$ Crawford+ (1988), 10.1016/0019-1035(88)90085-1; ${ }^{10}$ Muñoz-Iglesias+ (2013), 10.1089/ast.2012.0900; ${ }^{11} \mathrm{Neveu}+$ (2014), 10.1016/j.icarus.2014.03.043; ${ }^{12}$ Pappalardo+ (1996), LPSC 27; ${ }^{13}$ Greenberg+ 
(1998), 10.1006/icar.1998.5986; ${ }^{14}$ Tufts + (2000), 10.1006/icar.2000.6369; $;{ }^{15}$ Dombard + (2013), 10.1016/j.icarus.2012.11.021; ${ }^{16}$ Walker+ (2015), 10.1002/2014GL062405; ${ }^{17}$ Manga + (2017), 10.1016/j.icarus.2016.10.009; ${ }^{18}$ Neveu + (2015), 10.1002/2015GL066375; ${ }^{19}$ Quick + (2016), 10.1016/j.jvolgeores.2016.03.018; ${ }^{20} \mathrm{Craft}+(2018)$, Cryovolcanism in the Solar System Workshop; ${ }^{21}$ Quick + (2019), 10.1016/j.icarus.2018.07.016; ${ }^{22}$ Lesage + (2020), 10.1016/j.icarus.2019.07.003; ${ }^{23}$ Kalousová+ (2016), 10.1002/2016JE005188; ${ }^{24} \mathrm{Hammond}+$ (2018), 10.1029/2018JE005781; ${ }^{{ }^{2} 5}$ Schmidt+ (2020), Dive, dive, dive: [...]; ${ }^{26}$ Lucchita (1980), 10.1016/0019-1035(80)90039-1; ${ }^{27}$ Showman + (2004), 10.1016/j.icarus.2004.07.011; ${ }^{28}$ Porco+ (2006), 10.1126/science.1123013; ${ }^{29}$ Soderblom + (1990), 10.1126/science.250.4979.410; ${ }^{30}$ Roth + (2013), 10.1126/science.1247051; ${ }^{31}$ Sparks + (2016), 10.3847/0004-637X/829/2/121; ${ }^{32}$ Sparks + (2017),10.3847/2041-8213/aa67f8; ${ }^{33}$ Jankowski+ (1988), 10.1126/science.241.4871.1322; ${ }^{34}$ Elachi+ (2005), 10.1126/science.1109919; ${ }^{35}$ Lopes + (2007), 10.1016/j.icarus.2006.09.006; ${ }^{36}$ Lopes + (2013), 10.1002/jgre.20062; ${ }^{37}$ Wood + (2020), 10.1029/2019JE006036; ${ }^{38}$ Buczkowski+ (2016), 10.1126/science.aaf4332; ${ }^{39}$ Gaidos (2001), 10.1006/icar.2001.6649; ${ }^{40}$ Michaut + (2014), 10.1002/2013JE004558; ${ }^{41}$ Moore+ (2016), 10.1126/science.aad7055; ${ }^{42}$ Porco + (2017), 10.1089/ast.2017.1665; ${ }^{43}$ Khurana + (1998), 10.1038/27394; ${ }^{44}$ Reynolds + (1979), 10.1029/GL006i002p00121; ${ }^{45}$ Pappalardo + (2009), 10.1111/j.1945-5100.2011.01193.x; ${ }^{46}$ Quick + (2017), 10.1016/j.icarus.2016.06.029; ${ }^{47}$ Buratti + (1988), 10.1016/0019-1035(88)90156-X; ${ }^{48}$ Schmidt + (2011), 10.1038/nature10608; ${ }^{49}$ Walker + (2017), LPSC 48(2824); ${ }^{50}$ Greeley+ (2000), 10.1029/1999JE001173; ${ }^{51} \mathrm{Hand}+(2015)$, 10.1002/2015GL063559; ${ }^{52}$ Waite + (2006),10.1126/science.1121290; ${ }^{{ }^{5} 53} \mathrm{Neveu}+(2020)$,

Returning samples from Enceladus for life detection; ${ }^{54} \mathrm{Hedman}+(2013), 10.1038 /$ nature12371; ${ }^{55}$ Ingersoll + (2020), 10.1016/j.icarus.2019.06.006; ${ }^{56}$ Hurford + (2007), 10.1038/nature05821; ${ }^{57} \mathrm{Hsu}+$ (2015), 10.1038/nature14262; ${ }^{58}$ Spencer+ (2006), 10.1126/science.1121661;

${ }^{59}$ Nakajima+ (2016), 10.1016/j.icarus.2016.02.027; ${ }^{60} \mathrm{Nixon}+(2018), 10.1016 / \mathrm{j}$. pss.2018.02.009; ${ }^{61}$ Sotin + (2005), 10.1038/nature03596; ${ }^{62}$ Moore + (2011), 10.1016/j.icarus.2011.01.019;

${ }^{63}$ Solomonidou + (2018), 10.1002/2017JE005477; ${ }^{64} \mathrm{Neish}+$ (2018), 10.1089/ast.2017.1758; ${ }^{65}$ McKinnon+ (2007), 10.1016/B978-012088589-3/50030-X; ${ }^{66}$ Schenk (1992), LPSC 23 (1215); ${ }^{67}$ Hansen+ (2015), LPSC 46; ${ }^{68}$ Schenk+ (1993), 10.1130/0091-7613(1993)021; ${ }^{{ }^{60}}$ Hansen+ (2020), Triton: Compelling Destination!; ${ }^{70}$ Singer+ (2018), 10.1016/j.icarus.2018.01.012; ${ }^{71}$ Cruikshank + (2019), 10.1016/j.icarus.2019.04.023; ${ }^{72}$ Cruikshank+ (2019), 10.1089/ast.2018.1927; ${ }^{73}$ Conrad+ (2016), AGU (P44A-05); ${ }^{74}$ Martin+ (2020), 10.1016/j.icarus.2020.113763; ${ }^{75}$ Keane + (2016), 10.1038/nature20120; ${ }^{76} \mathrm{Nimmo}+(2016)$, 10.1038/nature20148; ${ }^{77}$ Beyer+ (2019), 10.1016/j.icarus.2018.12.036; ${ }^{78}$ Desch + (2017), 10.1016/j.icarus.2016.11.037; ${ }^{{ }^{\circ} 79} \mathrm{Holler}+$ (2020), Future Exploration of the Trans-Neptunian Region; ${ }^{{ }^{800}}$ Robbins+ (2020), Pluto System Follow On Missions; ${ }^{8}{ }^{81}$ Howett + (2020), Persephone [...] Mission Concept; ${ }^{8} 82$ Runyon+ (2020), Comparative Planetology of Kuiper Belt Dwarf Planets; ${ }^{83}$ Russell+ (2016), 10.1126/science.aaf4219; ${ }^{8} 84$ Castillo-Rogez+ (2020), Science Motivations for the Future Exploration of Ceres; ${ }^{85}$ Stein + (2019), 10.1016/j.icarus.2017.10.014; ${ }^{86}$ Sori + (2018), 10.1038/s41550-018-0574-1; ${ }^{87}$ Raponi + (2019), 10.1016/j.icarus.2018.02.001; ${ }^{88}$ Carrozzo+ (2018), 10.1126/sciadv.1701645; ${ }^{89} \mathrm{Scully}+$ (2019), 10.1016/j.icarus.2018.08.029; ${ }^{90}$ Buczkowski+ (2018), 10.1029/2018JE005632; ${ }^{91}$ Ruesch + (2016), 10.1126/science.aaf4286; ${ }^{92}$ Bland + (2019), 10.1038/s41561-019-0453-0; ${ }^{93}$ Jozwiak + (2012), 10.1029/2012JE004134; ${ }^{94}$ Schultz (1976), 10.1007/BF00562240; ; ${ }^{95}$ Rivera-Valentín+ (2020), Who is Missing in Planetary Science?: Demographics [...]; ${ }^{996}$ Rathbun $+(2020)$, Who is Missing in Planetary

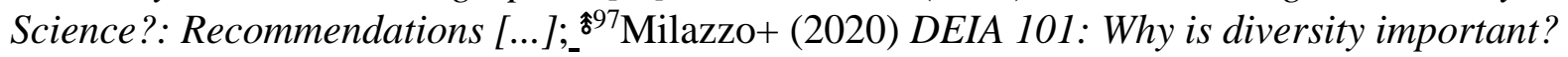

